University of Nebraska - Lincoln

DigitalCommons@University of Nebraska - Lincoln

Faculty Publications from the Department of Engineering Mechanics

2009

\title{
Harvesting magnetic energy using extensional vibration of laminated magnetoelectric plates
}

C. L. Zhang

Zhejiang University, Hangzhou, PRC

J. S Yang

University of Nebraska-Lincoln, jyang1@unl.edu

W. Q. Chen

Zhejiang University, Hangzhou, PRC

Follow this and additional works at: https://digitalcommons.unl.edu/engineeringmechanicsfacpub

Part of the Mechanical Engineering Commons

Zhang, C. L.; Yang, J. S; and Chen, W. Q., "Harvesting magnetic energy using extensional vibration of laminated magnetoelectric plates" (2009). Faculty Publications from the Department of Engineering Mechanics. 65.

https://digitalcommons.unl.edu/engineeringmechanicsfacpub/65

This Article is brought to you for free and open access by the Mechanical \& Materials Engineering, Department of at DigitalCommons@University of Nebraska - Lincoln. It has been accepted for inclusion in Faculty Publications from the Department of Engineering Mechanics by an authorized administrator of DigitalCommons@University of Nebraska - Lincoln. 


\title{
Harvesting magnetic energy using extensional vibration of laminated magnetoelectric plates
}

\author{
C. L. Zhang, ${ }^{1}$ J. S. Yang, ${ }^{2}$ and W. Q. Chen ${ }^{3, a)}$ \\ ${ }^{1}$ Department of Civil Engineering, Zhejiang University, Hangzhou 310058, People's Republic of China \\ ${ }^{2}$ Department of Engineering Mechanics, University of Nebraska, Lincoln, Nebraska 68588-0526, USA \\ ${ }^{3}$ Department of Engineering Mechanics, Zhejiang University, Hangzhou 310027, People's Republic of China
}

(Received 15 June 2009; accepted 19 June 2009; published online 10 July 2009)

\begin{abstract}
Magnetically forced extensional vibrations of laminated plates with piezoelectric and piezomagnetic layers are analyzed theoretically. It is shown that such a structure can be used to harvest magnetic energy and convert it to electric energy. The output power and the energy conversion efficiency are calculated. The load dependence of the magnetoelectric coupling coefficient is also obtained. (C) 2009 American Institute of Physics. [DOI: 10.1063/1.3176981]
\end{abstract}

Piezoelectric and piezomagnetic materials are common transducer materials. ${ }^{1}$ They used to be used separately. Recently, various composites and laminates of piezoelectric and piezomagnetic materials have been developed. ${ }^{2,3}$ In these materials and structures, electric and magnetic, as well as acoustic fields all couple together. From a magnetoelectric (ME) point of view, separate electric and magnetic behaviors of each phase couple through mechanical fields. Strong ME couplings have been demonstrated. ${ }^{4-6}$ Both static ${ }^{7,8}$ and frequency dependent ${ }^{9-12}$ ME couplings have been studied experimentally and theoretically. Potentially these new materials and structures can be used for magnetic field sensors, current sensors, energy harvesters, transformers, and ME filters, as well as phase shifters. ${ }^{2}$ An energy harvester for collecting vibration energy and converting it to magnetic and then electric energy using ME materials was described in Ref. 2. In this paper we analyze theoretically using ME materials for collecting magnetic energy and converting it to electric energy. We study time-harmonic vibrations of a laminated ME plate of piezoelectric and piezomagnetic layers driven by a magnetic field. It is shown that the device can operate as a power harvester for applications in a magnetic environment, or as part of the mechanical energy harvester in Ref. 2.

Consider the laminated plate shown in the inset of Fig. 1. We use polarized ceramics PZT-4 (a kind of lead zirconate titanate or PZT) for the piezoelectric layer, and $\mathrm{CoFe}_{2} \mathrm{O}_{4}$ for the piezomagnetic layers. The poling direction of the PZT-4 layer is denoted by $\mathrm{P}$ in the inset. $\mathrm{CoFe}_{2} \mathrm{O}_{4}$ is magnetostrictive and appears piezomagnetic under a dc biasing magnetic field whose direction is indicated by $\mathrm{M}$. The relevant material constants can be found in Refs. 13 and 14. We consider the so-called cylindrical or plane-strain motion of the plate, and a unit width of the plate in the $x_{2}$ direction is taken. The electrodes on the piezoelectric layer are at the left and right ends. They are connected by a circuit whose impedance in time-harmonic motions is denoted by $Z$, which is the load impedance. There is an applied magnetic field in the normal direction of the plate such that in the piezomagnetic layers we have $H_{1}=H_{2}=0$ and $H_{3}=H \exp (\mathrm{i} \omega t)$. For steady-state motions, we use the usual complex notation with all fields having the same time dependence, which will be dropped.

${ }^{a)}$ Electronic mail: chenwq@zju.edu.cn.
We use the recently derived equations for laminated plates of piezoelectric and piezomagnetic layers. ${ }^{15}$ Let the plate middle-plane extensional displacement be $u_{1}\left(x_{1}, t\right)$, the longitudinal distribution of the electric potential be $\phi\left(x_{1}, t\right)$, the extensional resultant force be $N$, and the longitudinal electric displacement resultant be $D$. We consider a plate with free ends. Then ${ }^{15}$

$$
\begin{aligned}
& N_{, 1}=c_{11}^{(0)} u_{1,11}+e_{11}^{(0)} \phi_{, 11}=-\omega^{2} \rho^{(0)} u_{1}, \quad\left|x_{1}\right|<a, \\
& D_{, 1}=e_{11}^{(0)} u_{1,11}-\varepsilon_{11}^{(0)} \phi_{, 11}=0, \quad\left|x_{1}\right|<a, \\
& N=c_{11}^{(0)} u_{1,1}+e_{11}^{(0)} \phi_{, 1}-h_{31}^{(0)} H=0, \quad x_{1}= \pm a,
\end{aligned}
$$

where Eq. (1) is for motion for extension, Eq. (2) is for electrostatics, and Eq. (3) is for mechanical boundary conditions. The magnetic field is given. Therefore no equation is needed for it. The effective plate material constants in Eqs. (1)-(3) are related to the three-dimensional material constants through

$$
\begin{aligned}
& \rho^{(0)}=2 \rho^{\mathrm{I}}\left(h-h^{\prime}\right)+2 \rho^{\mathrm{II}} h^{\prime}, \\
& c_{11}^{(0)}=2\left(h-h^{\prime}\right) \bar{c}_{11}^{\mathrm{I}}+2 h^{\prime} \bar{c}_{11}^{\mathrm{II}}, \\
& h_{31}^{(0)}=\bar{h}_{31}^{\mathrm{I}} 2\left(h-h^{\prime}\right), \quad e_{11}^{(0)}=\bar{e}_{11}^{\mathrm{II}} 2 h^{\prime}, \quad \varepsilon_{11}^{(0)}=\bar{\varepsilon}_{11}^{\mathrm{II}} 2 h^{\prime},
\end{aligned}
$$

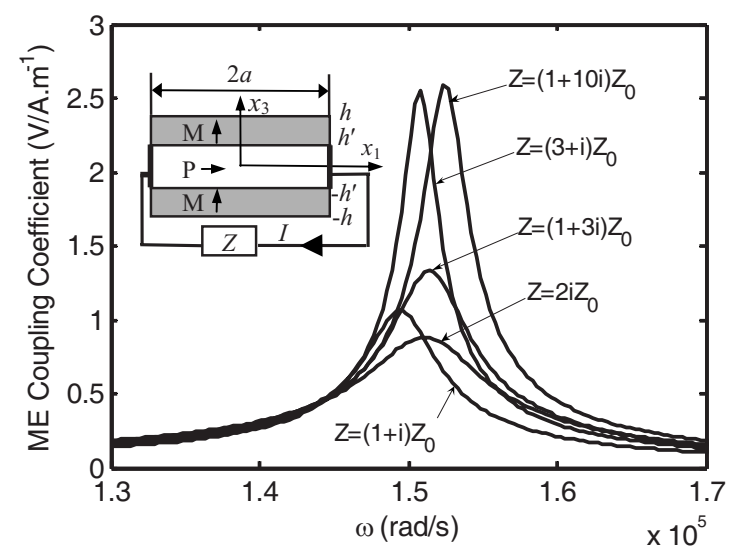

FIG. 1. ME coefficient vs driving frequency of the laminated piezoelectric/ piezomagnetic plate shown as the inset. 


$$
\begin{array}{ll}
\bar{c}_{11}^{\mathrm{I}}=c_{11}^{\mathrm{I}}-\left(c_{13}^{\mathrm{I}}\right)^{2} / c_{33}^{\mathrm{I}}, & \bar{h}_{31}^{\mathrm{I}}=h_{31}^{\mathrm{I}}-h_{33}^{\mathrm{I}} c_{31}^{\mathrm{I}} / c_{33}^{\mathrm{I}}, \\
\bar{c}_{11}^{\mathrm{II}}=c_{33}^{\mathrm{II}}-\left(c_{13}^{\mathrm{II}}\right)^{2} / c_{11}^{\mathrm{II}}, & \bar{e}_{11}^{\mathrm{II}}=e_{33}^{\mathrm{II}}-e_{31}^{\mathrm{II}} c_{13}^{\mathrm{II}} / c_{11}^{\mathrm{II}}, \\
\bar{\varepsilon}_{11}^{\mathrm{II}}=\varepsilon_{33}^{\mathrm{II}}+\left(e_{31}^{\mathrm{II}}\right)^{2} / c_{11}^{\mathrm{II}} . &
\end{array}
$$

In Eqs. (4) and (5), the superscripts "I" and "II" are for the piezomagnetic and piezoelectric layers, respectively. The charge on the right electrode is given by $Q_{e}=-D$ $=-\left(e_{11}^{(0)} u_{1,1}-\varepsilon_{11}^{(0)} \phi_{, 1}\right)$ and the electric current flowing out of this electrode is

$$
I=-\dot{Q}_{e}=i \omega\left(e_{11}^{(0)} u_{1,1}-\varepsilon_{11}^{(0)} \phi_{, 1}\right), \quad x_{1}=a .
$$

For the circuit joining the two electrodes we have

$$
I=[\phi(a)-\phi(-a)] / Z \text {. }
$$

Equations (1)-(3) and (7) form a well-defined mathematical problem. Its solution can be readily obtained as

$$
u_{1}=C_{1} \sin k x_{1}, \quad \phi=\frac{e_{11}^{(0)}}{\varepsilon_{11}^{(0)}} C_{1} \sin k x_{1}+C_{2} x_{1},
$$

where $C_{1}$ and $C_{2}$ are undetermined constants, and $k^{2}$ $=\omega^{2} \rho^{(0)} / \hat{c}_{11}^{(0)}$ with $\hat{c}_{11}^{(0)}=c_{11}^{(0)}+\left(e_{11}^{(0)}\right)^{2} / \varepsilon_{11}^{(0)}$. Substituting Eq. (8) into Eqs. (3) and (7) determines $C_{1}$ and $C_{2}$.

The ME voltage coupling coefficient is defined by and can be calculated from

$$
r=\frac{\phi(a)-\phi(-a)}{H} .
$$

The average output electrical power over a period is given by $P_{e}=|I|^{2} \operatorname{Re}(Z) / 2$, here the operation "Re" is for taking the real part of a complex number. The relevant magnetic flux component $B_{3}$ in the piezomagnetic layers is determined from $^{15}$

$$
B_{3}=\frac{1}{2\left(h-h^{\prime}\right)}\left(h_{31}^{(0)} u_{1,1}+\mu_{33}^{(0)} H\right),
$$

where $\mu_{33}^{(0)}=\bar{\mu}_{33}^{\mathrm{I}} 2\left(h-h^{\prime}\right)$ and $\bar{\mu}_{33}^{\mathrm{I}}=\mu_{33}^{\mathrm{I}}+\left(h_{33}^{\mathrm{I}}\right)^{2} / c_{33}^{\mathrm{I}}$.

The input magnetic power averaged over a period is calculated from

$$
\begin{aligned}
P_{m}= & 2\left(h-h^{\prime}\right) \int_{-a}^{a} d x_{1} \frac{1}{T} \\
& \times \int_{0}^{T} \operatorname{Re}[H \exp (i \omega t)] \operatorname{Re}\left[i \omega B_{3} \exp (i \omega t)\right] d t,
\end{aligned}
$$

where $T=2 \pi / \omega$. The efficiency $\eta$ of the energy conversion and the power density $p$ of the device are defined by

$$
\eta=\frac{P_{e}}{P_{m}}, \quad p=\frac{P_{e}}{4 a h} .
$$

We use $Z_{0}=a /\left(\mathrm{i} h^{\prime} \omega \varepsilon_{33}\right)$ in the following as a unit for measuring the load impedance $Z$. $Z_{0}$ is related to the capacitance of the piezoelectric layer. Material damping is described by complex elastic constants with their imaginary parts $1 \%$ of their real parts. A unit driving magnetic field of $H=1 \mathrm{~A} / \mathrm{m}$ in SI units is used. For dimensions we consider $2 h=0.7 \mathrm{~cm}, 2 a=9.2 \mathrm{~cm}$, and $h^{\prime}=0.6 h$.

First we examine the ME coupling coefficient $|r|$. Its frequency dependence is shown in Fig. 1 which is typical,

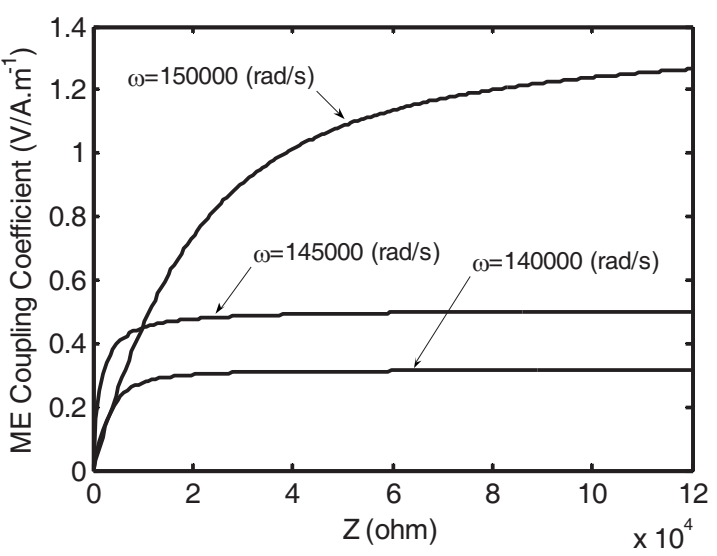

FIG. 2. ME coefficient vs load impedance ( $Z$ real).

with peaks at resonance. Only the first resonance is shown. At higher resonances the modes have nodal points which lower the output voltage and are of less interest. The exact locations of the peaks depend on the load. One of the main results of this work is the load dependence of the ME coefficient near the first resonance shown in Fig. 2. For small loads the electrodes are nearly shorted. The coefficient begins from zero and increases essentially linearly for small loads. For large loads the electrodes are essentially open and the coefficient saturates.

The behavior of the output power density versus the load impedance is depicted in Fig. 3, which shows that there is a maximum corresponding to a particular load. Therefore proper design of the output circuit called impedance match is needed for maximal output. The locations of the maxima in Fig. 3 are sensitive to frequency. The dependence of the output power density on frequency is similar to that shown in Fig. 2, indicating that the device can only produce significant output near resonance.

Efficiency versus load impedance is shown in Fig. 4. It is seen that the efficiency has a maximum for a particular load. The efficiency is quite high, about $80 \%$ at its optimal value. The locations of the maxima are insensitive to frequency. Also, the similar frequency dependence indicates that the efficiency is large near resonance.

In summary, we have analyzed and observed the load dependence of the ME coefficient $|r|$ and the basic behaviors of the power density and efficiency. The output power and efficiency are relatively large near resonance. They are sensitive to the impedance of the output circuit and there exist

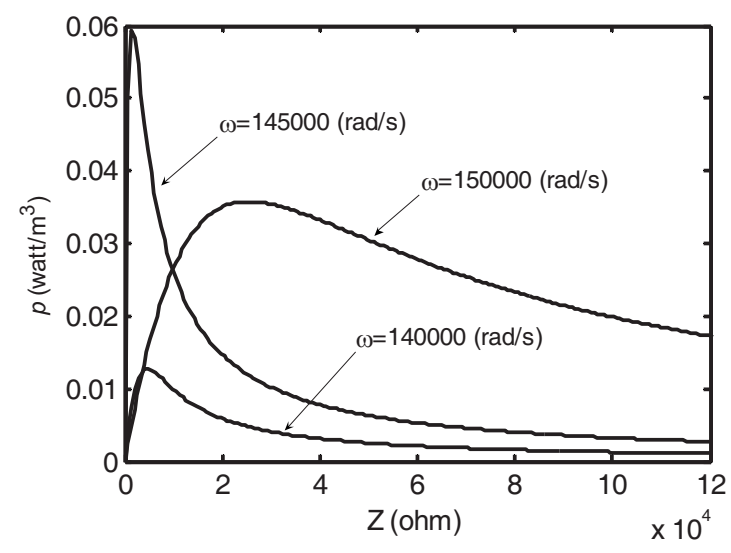

FIG. 3. Power density vs load impedance ( $Z$ real). 


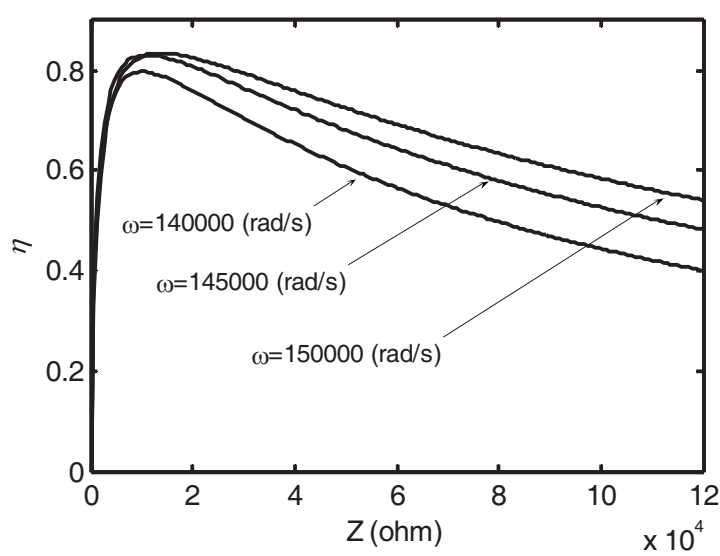

FIG. 4. Efficiency vs load impedance ( $Z$ real).

optimal values of the impedance for maximal output power or efficiency. These results are basic to the understanding and development of ME energy harvesters. At present there are no results from experiment or other analyses to compare with.

The work was supported by the NSFC (Grant Nos. 10725210 and 10832009), the Specialized Research Fund for the Doctoral Program of Higher Education (Grant No.
20060335107), and the National Basic Research Program of China (Grant No. 2009CB623200).

${ }^{1}$ A. Ballato, IEEE Trans. Ultrason. Ferroelectr. Freq. Control 48, 1189 (2001).

${ }^{2}$ S. Priya, R. Islam, S. X. Dong, and D. Viehland, J. Electroceram. 19, 147 (2007).

${ }^{3}$ C.-W. Nan, M. I. Bichurin, S. X. Dong, D. Viehland, and G. Srinivasan, J. Appl. Phys. 103, 031101 (2008).

${ }^{4}$ S. X. Dong, J. G. Bai, J. Y. Zhai, J.-F. Li, G.-Q. Lu, and D. Viehland, Appl. Phys. Lett. 86, 182506 (2005).

${ }^{5}$ J. Y. Zhai, Z. P. Xing, S. X. Dong, J. F. Li, and D. Viehland, Appl. Phys. Lett. 88, 062510 (2006).

${ }^{6}$ J. Y. Zhai, S. X. Dong, Z. P. Xing, J. F. Li, and D. Viehland, Appl. Phys. Lett. 91, 123513 (2007).

${ }^{7}$ S. Dong, J.-F. Li, and D. Viehland, IEEE Trans. Ultrason. Ferroelectr. Freq. Control 50, 1236 (2003).

${ }^{8}$ Y.-M. Jia, F.-F. Wang, X.-Y. Zhao, H.-S. Luo, S. W. Or, and H. L. W. Chan, Compos. Sci. Technol. 68, 1440 (2008).

${ }^{9}$ M. I. Bichurin, D. A. Filippov, and V. M. Petrov, Phys. Rev. B 68, 132408 (2003).

${ }^{10}$ Z. P. Xing, S. X. Dong, J. Y. Zhai, L. Yan, J. F. Li, and D. Viehland, Appl. Phys. Lett. 89, 112911 (2006).

${ }^{11}$ N. Cai, Y. Zhao, X. H. Geng, and S. W. Or, J. Alloys Compd. 448, 89 (2008).

${ }^{12}$ Z. Shi, J. Ma, and C.-W. Nan, J. Electroceram. 21, 390 (2008).

${ }^{13}$ F. Ramirez, P. R. Heyliger, and E. N. Pan, J. Sound Vib. 292, 626 (2006).

${ }^{14}$ J. H. Huang, Y.-H. Chiu, and H.-K. Liu, J. Appl. Phys. 83, 5364 (1998).

${ }^{15}$ C. L. Zhang, W. Q. Chen, J. Y. Li, and J. S. Yang, IEEE Trans. Ultrason. Ferroelectr. Freq. Control 56, 1046 (2009). 